\title{
QUALIDADE DOS REGISTROS DA ASSISTÊNCIA PRÉ-NATAL NA CADERNETA DA GESTANTE
}

\author{
QUALITY OF THE PRENATAL CARE RECORDS \\ IN THE PREGNANT WOMEN'S BOOKLET
}

\section{CALIDAD DE LOS RECORDADOS DE CUIDADO PRENATAL EN EL CUADERNO DE LA MUJER PREGNANT}

\author{
Thaíse Almeida Rodrigues ${ }^{1}$ \\ Ana Karina Bezerra Pinheiro ${ }^{2}$ \\ Andressa Arraes Silva ${ }^{3}$ \\ Luciene Rocha Garcia Castro ${ }^{4}$ \\ Milka Borges da Silva ${ }^{5}$ \\ Lena Maria Barros Fonseca ${ }^{6}$
}

Como citar este artigo: Rodrigues TA, Pinheiro AKB, Silva AA, Castro LRG, Silva MB, Fonseca LMB. Qualidade dos registros da assistência pré-natal na caderneta da gestante. Rev baiana enferm. 2020;34:e35099.

Objetivo: avaliar a qualidade dos registros na caderneta da gestante averiguando a legibilidade e completude dos registros da assistência pré-natal de risco habitual. Método: estudo avaliativo, descritivo e quantitativo, realizado em nove unidades de saúde da atenção básica em São Luís, Maranhão, Brasil, no período de maio de 2017 a setembro de 2018. Os registros foram avaliados quanto à legibilidade e completude. Resultados: 92,4\% das cadernetas foram consideradas com registros legíveis. A avaliação da completude evidenciou que $72,4 \%$ apresentaram completude ruim. Nenhuma caderneta foi classificada com registro bom ou excelente. A avaliação por seções demonstrou que a seção de Exames Complementares de Rotina apresentou os melhores resultados, enquanto a seção de Atividades Complementares apresentou os menores níveis de completude. Conclusão: os registros nas cadernetas da gestante foram predominantemente ruins, denotando uma desvalorização desse instrumento e um provável descumprimento das recomendações do Ministério da Saúde para uma assistência de qualidade.

Descritores: Avaliação em Saúde. Qualidade da Assistência à Saúde. Assistência Pré-natal. Registros de Saúde Pessoal.

Objective: to evaluate the quality of the records in the pregnant women's booklet, assessing the legibility and completeness of usual-risk pre-natal records. Method: evaluative, descriptive and quantitative study, conducted in nine basic health care units in São Luis, Maranhão, Brazil, in the period from May 2017 to September 2018. The records were evaluated according to legibility and completeness. Results: $92.4 \%$ of the booklets were considered with legible records. The assessment of the completeness showed that $72.4 \%$ had bad completeness. No record booklet was classified as good or excellent. The assessment by sections showed that the Routine Complementary Test section showed the best results, whereas the Complementary Activities section showed the lowest levels of completeness. Conclusion: the records in the pregnant women's booklet were predominantly bad, denoting a devaluation of this instrument and a probable noncompliance with the recommendations of the Ministry of Health for a quality assistance.

Descriptors: Health Evaluation. Quality of Health Care. Prenatal Care. Health Records, Personal.

\footnotetext{
Enfermeira. Mestre em Enfermagem. Professora Assistente da Faculdade de Imperatriz. Imperatriz, Maranhão, Brasil. thaisealmeidaguimaraes@hotmail.com. https:// orcid.org/0000-0003-2138-5934.

Enfermeira. Doutora em Enfermagem. Professora da Universidade Federal do Ceará. Fortaleza, Ceará, Brasil. https://orcid.org/0000-0003-3837-4I31 .

Enfermeira. Mestre em Enfermagem. Professora Substituta da Universidade Estadual do Maranhão. Bacabal, Maranhão, Brasil. https://orcid.org/0000-0002-1699-5597.

Enfermeira. Pesquisadora independente. São Luís, Maranhão, Brasil. https://orcid.org/0000-0002-1243-9737.

Enfermeira. Pesquisadora independente. São Luís, Maranhão, Brasil. https://orcid.org/0000-0002-3704-255X.

Enfermeira. Doutora em Biotecnologia. Professor Associado da Universidade Federal do Maranhão. São Luís, Maranhão, Brasil. https://orcid.org/0000-0002-6499-I005.
} 
Objetivo: evaluar la calidad de los registros en la cartilla de las mujeres embarazadas, evaluando la legibilidad y la integridad de los registros de control prenatal de riesgo habitual. Método: estudio evaluativo, descriptivo y cuantitativo, realizado en nueve unidades de atención básica en salud en São Luís, Maranhão, Brasil, en el periodo de mayo de 2017 a septiembre de 2018. Los registros fueron evaluados en cuanto a la legibilidad y la integridad. Resultados: el 92,4\% de las cartillas fueron consideradas con registros legibles. La evaluación de la integridad mostró que el 72,4\% tenía mala integridad. Ningún registro de la cartilla fue clasificado como bueno o excelente. La evaluación por secciones mostró que la sección de Exámenes Complementarios de Rutina obtuvo los mejores resultados, mientras que la sección de Actividades Complementarias obtuvo los niveles más bajos de la integridad. Conclusión: los registros de la cartilla de mujeres embarazadas eran, en su mayoría, pobres, denotando una devaluación de este instrumento y un probable incumplimiento de las recomendaciones del Ministerio de Salud para una asistencia de calidad.

Descriptores: Evaluación en Salud. Calidad de la Atención de Salud. Atención Prenatal. Registros de Salud Personal.

\section{Introdução}

A assistência pré-natal adequada constitui considerável determinante dos indicadores de saúde relacionados à mãe e ao bebê, apresentando o potencial de minimizar as principais causas de mortalidade materna e infantil ${ }^{(1)}$. No contexto brasileiro, o Ministério da Saúde (MS) proporciona um conjunto de recomendações no intuito de qualificar a atenção pré-natal, dentre as quais estão o início precoce, periódico e contínuo, a realização de exames laboratoriais, a classificação de risco gestacional, a realização de práticas educativas e o registro das informações em prontuário e no cartão da gestante ${ }^{(2)}$.

O cartão da gestante, criado na década de 1980, foi instituído como um dos instrumentos de registro da assistência pré-natal, deve conter os principais dados de acompanhamento da gestação e permanecer sempre com a gestante, facilitando, assim, o fluxo de informações entre os serviços de assistência à mulher no ciclo gravídico-puerperal $^{(3)}$. Ao longo dos anos, algumas reformulações foram realizadas, culminando com a publicação da caderneta da gestante em 2014, como parte das ações da estratégia Rede Cegonha para qualificação da atenção à saúde da mulher na gestação, parto e puerpério ${ }^{(4)}$.

A caderneta da gestante ampliou o papel do cartão da gestante, na medida em que incluiu um conjunto de orientações e procedimentos de ampla importância na atenção pré-natal que, até então, não eram contemplados, como informações sobre as modificações do organismo materno e o desenvolvimento do bebê, dicas para uma gravidez saudável e sinais de alerta, orientações sobre trabalho de parto e parto, sugestões para o sucesso da amamentação, e espaço para o registro de atividades educativas, consulta odontológica e pré-natal do parceiro ${ }^{(5)}$.

Desde sua implantação, esse instrumento tem sido considerado uma importante ferramenta de comunicação entre profissionais, permitindo a continuidade da assistência em todos os níveis de atenção à saúde. Além da função no sistema de referência e contrarreferência, atualmente tem sido utilizado como ferramenta de avaliação dos serviços de saúde, tendo em vista que a qualidade dos registros realizados pode ser um reflexo da qualidade da assistência prestada, pois, uma vez que um procedimento é comprovadamente benéfico, a simples presença ou ausência de registros desse procedimento pode ser considerada como indicativo de boa ou má qualidade ${ }^{(6-8)}$.

Estudos realizados em municípios de diferentes regiões do Brasil evidenciaram baixos níveis de completude dos registros no cartão da gestante, o que demonstra que muitos procedimentos estão deixando de ser realizados e/ ou registrados. Além disso, quando registrados, muitos demonstram inadequação aos parâmetros preconizados pelo Ministério da Saúde ${ }^{(7,9-11)}$.

Provavelmente em virtude da implantação relativamente recente da caderneta da gestante, poucos estudos de avaliação dos registros nessa versão foram publicados. Diante disso, esta pesquisa teve como objetivo avaliar a qualidade dos 
registros na caderneta da gestante, averiguando a legibilidade e completude dos registros da assistência pré-natal de risco habitual em um município do nordeste brasileiro.

\section{Método}

Pesquisa avaliativa, descritiva, com abordagem quantitativa, realizada em unidades de saúde que realizam assistência pré-natal de risco habitual no município de São Luís, Maranhão, Brasil, no período de maio de 2017 a setembro de 2018. O município de São Luís é dividido em sete Distritos Sanitários, dos quais três foram selecionados para esta pesquisa: Centro, Bequimão e Itaqui-Bacanga. De cada Distrito Sanitário, foram selecionadas três unidades de saúde, totalizando nove unidades.

A população do estudo foi constituída por gestantes que receberam assistência pré-natal nas unidades de saúde selecionadas, no período da coleta de dados. Utilizou-se amostragem não probabilística, por conveniência, de acordo com a demanda dos serviços. Dessa forma, a amostra foi composta por 105 gestantes que atenderam aos seguintes critérios de inclusão: gestantes de risco habitual, após a $30^{\text {a }}$ semana de gestação, de qualquer idade, que apresentassem capacidade de comunicação, tivessem realizado, no mínimo, três consultas de pré-natal e estivessem portando a caderneta da gestante no momento da coleta de dados.

Para a coleta de dados foi utilizado um instrumento composto por um checklist correspondente aos campos para preenchimento constantes no atual modelo de caderneta da gestante padronizado pelo Ministério da Saúde ${ }^{(5)}$. Os itens foram considerados como "sim" quando o registro estava presente e completo, "parcial" quando o registro estava presente, mas incompleto, e "não" quando o registro estava ausente.

Os dados coletados foram inseridos em banco de dados por meio do software Epi Info 7. Posteriormente, os dados foram revisados para identificar possíveis erros de digitação. Os resultados foram apresentados por meio de frequências absolutas e relativas.
Os registros foram classificados como legíveis, ilegíveis ou parcialmente legíveis. A avaliação da completude ocorreu por meio de parâmetro preconizado na literatura ${ }^{(12-13)}$. Para cada item da caderneta, a verificação da completude foi realizada dividindo-se o total de ausência de informação do item pelo total da amostra e, em seguida, realizada a classificação segundo os níveis de qualidade: excelente (incompletude $<5 \%$ ), bom $(5 \% \leq$ incompletude $<10 \%$ ), regular $(10 \% \leq$ incompletude $<20 \%)$, ruim $(20 \%$ $\leq$ incompletude $<50 \%$ ) e muito ruim (incompletude $\geq 50 \%$ ). Os mesmos critérios foram considerados para classificar cada caderneta da gestante, ponderando a divisão do total de itens não preenchidos pelo total de itens disponíveis para registro.

Para realizar a classificação da completude de cada item da caderneta, foram estabelecidas sete seções: Identificação; Classificação de Risco Gestacional; Gráficos de Acompanhamento e Suplementação Vitamínica; Procedimentos Gineco-obstétricos; Exames Complementares de Rotina; Imunização; e Atividades Complementares.

Este estudo é vinculado à pesquisa intitulada "Retratando a assistência pré-natal em São Luís MA", a qual obteve parecer favorável do Comitê de Ética em Pesquisa (CEP) da Universidade Federal do Maranhão (UFMA), sob n. 1.999.550, em 4 de abril de 2017. A pesquisa seguiu as recomendações da Resolução n. 466/2012 do Conselho Nacional de Saúde (CNS). As gestantes que atenderam aos critérios de inclusão receberam informações acerca da pesquisa e foram convidadas a participar. Após anuência e assinatura do Termo de Consentimento Livre e Esclarecido (TCLE) ou Termo de Assentimento (TA), a coleta de dados foi realizada individualmente, respeitando a privacidade das gestantes.

\section{Resultados}

Verificou-se que $92,4 \%$ das cadernetas da gestante foram consideradas com registros legíveis, $5,7 \%$ com registros parcialmente legíveis e 1,9\% com registros ilegíveis. 
A completude dos itens da seção de Identificação variou de 3,8\% (muito ruim) a 99,1\% (excelente), correspondendo ao número do Sistema de Acompanhamento do Programa de Humanização no Pré-Natal e Nascimento da Gestante (Sisprenatal) e nome, respectivamente. Nessa seção, houve predominância de itens com registro ruim e muito ruim, conforme apresentado na Tabela 1.

Tabela 1 - Completude dos registros de identificação nas cadernetas da gestante. São Luís, Maranhão, Brasil - maio 2017- set. $2018(\mathrm{~N}=105)$

\begin{tabular}{l|c|c|c}
\hline Variáveis & $\begin{array}{c}\text { Sim } \\
\mathbf{n}(\mathbf{\%})\end{array}$ & $\begin{array}{c}\text { Não } \\
\mathbf{n}(\mathbf{\%})\end{array}$ & Classificação \\
\hline Há registro quanto à (ao): & & & \\
Unidade de saúde do pré-natal & $78(74,3)$ & $27(25,7)$ & Ruim \\
Serviço indicado para o parto & $50(47,6)$ & $55(52,4)$ & Muito ruim \\
Número do cartão do Sistema Único de Saúde & $79(75,2)$ & $26(24,8)$ & Ruim \\
Número do Sisprenatal* & $4(3,8)$ & $98(93,3)$ & Muito ruim \\
Número de Identificação Social** & $9(8,6)$ & $83(79,1)$ & Muito ruim \\
Nome & $104(99,1 \%)$ & $1(1,0)$ & Excelente \\
Como gosta de ser chamada & $38(36,2)$ & $67(63,8)$ & Muito ruim \\
Nome do companheiro (opcional) & $41(39,1)$ & $64(61,0)$ & Muito ruim \\
Data de nascimento & $96(91,4)$ & $9(8,6)$ & Bom \\
Idade & $93(88,6)$ & $12(11,4)$ & Regular \\
Raça & $72(68,6)$ & $33(31,4)$ & Ruim \\
Trabalho fora de casa & $62(59,1)$ & $43(41,0)$ & Ruim \\
Ocupação & $54(51,4)$ & $51(48,6)$ & Ruim \\
Endereço & $77(73,3)$ & $28(26,7)$ & Ruim \\
Telefone & $73(69,5)$ & $32(30,5)$ & Ruim \\
E-mail & $15(14,3)$ & $90(85,7)$ & Muito ruim \\
Contato para emergência & $53(50,3)$ & $52(49,5)$ & Ruim \\
\hline
\end{tabular}

Fonte: Elaboração própria.

Notas:

* Excluídas 3 cadernetas da edição de 2018, por não conter esse item na edição.

* Excluídas 13 cadernetas da edição de 2014, por não conter esse item na edição.

Os registros da seção de Classificação de Risco Gestacional foram predominantemente ruins, com completudes variando entre 31,4\% (muito ruim) e 90,5\% (bom). Dados quantitativos referentes a gestações anteriores, como o número de partos, o número de cesarianas ou o número de natimortos, obtiveram melhor completude. As informações referentes ao tipo de gravidez (única, gemelar, tripla ou mais) e ao planejamento da gestação (planejada ou não planejada) estão entre os registros com menor completude (Tabela 2).

Tabela 2 - Completude dos registros de classificação de risco gestacional nas cadernetas da gestante. São Luís, Maranhão, Brasil - maio 2017-set. 2018 (N=105)

(continua)

\begin{tabular}{l|c|c|c|c}
\hline \multicolumn{1}{c|}{ Variáveis } & $\begin{array}{c}\text { Sim } \\
\mathbf{n ~ ( \% )}\end{array}$ & $\begin{array}{c}\text { Não } \\
\mathbf{n}(\%)\end{array}$ & $\begin{array}{c}\text { Parcial } \\
\mathbf{n}(\mathbf{\%})\end{array}$ & Classificação \\
\hline Há registro quanto à (ao): & & & & Ruim \\
Instrução & $60(57,1)$ & $45(42,9)$ & - & Ruim \\
Estado civil & $63(60,0)$ & $42(40,0)$ & - & Ruim \\
Peso anterior & $65(61,9)$ & $40(38,1)$ & - & Regular \\
Altura & $87(82,9)$ & $18(17,1)$ & - & Muito ruim \\
Tipo de gravidez & $50(47,6)$ & $55(52,4)$ & - &
\end{tabular}


Tabela 2 - Completude dos registros de classificação de risco gestacional nas cadernetas da gestante. São Luís, Maranhão, Brasil - maio 2017-set. 2018 (N=105) (conclusão)

\begin{tabular}{l|c|c|c|c}
\hline \multicolumn{1}{c|}{ Variáveis } & $\begin{array}{c}\text { Sim } \\
\mathbf{n ( \% )}\end{array}$ & $\begin{array}{c}\text { Não } \\
\mathbf{n}(\%)\end{array}$ & $\begin{array}{c}\text { Parcial } \\
\mathbf{n}(\%)\end{array}$ & Classificação \\
\hline Há registro quanto à (ao): & & & & Muito ruim \\
Classificação de risco & $33(31,4)$ & $72(68,6)$ & - & Muito ruim \\
Gravidez planejada & $43(41,0)$ & $62(59,1)$ & - & Ruim \\
Antecedentes familiares & $67(63,8)$ & $36(34,3)$ & $2(1,9)$ & Bom \\
Gestações & $95(90,5)$ & $5(4,8)$ & $5(4,8)$ & Ruim \\
Antecedentes clínicos & $66(62,9)$ & $34(32,4)$ & $5(4,8)$ & Ruim \\
Gestação atual & $53(50,5)$ & $28(26,7)$ & $24(22,9)$ & \\
\hline
\end{tabular}

Fonte: Elaboração própria.

Nota: Sinal convencional utilizado:

- Dado numérico igual a zero não resultante de arredondamento.

A seção de Gráficos de Acompanhamento e Suplementação Vitamínica apresentou completude muito ruim para todos os itens. Verificou-se que $55,2 \%$ das cadernetas encontravam-se sem registros no gráfico de acompanhamento nutricional, 58,1\% sem registros na curva de altura uterina por idade gestacional, $60,0 \%$ sem registros de suplementação de sulfato ferroso, e 61,9\% sem registros de suplementação de ácido fólico.
Os registros da seção de Procedimentos Gineco-obstétricos obtiveram, em sua maioria, completude ruim, variando entre $17,1 \%$ (muito ruim) e 91,4\% (bom). Os componentes com melhor avaliação foram referentes à data da última menstruação (DUM) e a data provável do parto (DPP), enquanto os registros de Índice de Massa Corporal (IMC), edema e altura uterina apresentaram os menores níveis de completude (Tabela 3).

Tabela 3 - Completude dos registros dos procedimentos gineco-obstétricos nas cadernetas da gestante. São Luís, Maranhão, Brasil maio 2017-set. 2018 (N=105)

\begin{tabular}{l|c|c|c|c}
\multicolumn{1}{c|}{ Variáveis } & $\begin{array}{c}\text { Sim } \\
\mathbf{n}(\mathbf{\%})\end{array}$ & $\begin{array}{c}\text { Não } \\
\mathbf{n}(\mathbf{\%})\end{array}$ & $\begin{array}{c}\text { Parcial } \\
\mathbf{n}(\mathbf{\%})\end{array}$ & Classificação \\
\hline Há registro quanto à (ao): & & & & Bom \\
Data da última menstruação & $95(90,5)$ & $10(9,5)$ & - & Bom \\
Data provável do parto & $96(91,4)$ & $9(8,6)$ & - & Ruim \\
Queixa & $59(56,2)$ & $3(2,9)$ & $43(41,0)$ & Regular \\
Idade gestacional & $89(84,8)$ & $0(0,0)$ & $16(15,2)$ & Regular \\
Peso & $89(84,8)$ & $0(0,0)$ & $16(15,2)$ & Muito ruim \\
Índice de massa corporal & $18(17,1)$ & $43(41,0)$ & $44(41,9)$ & Muito ruim \\
Edema & $40(38,1)$ & $10(9,5)$ & $55(52,4)$ & Regular \\
Pressão arterial & $86(81,9)$ & $0(0,0)$ & $19(18,1)$ & Muito ruim \\
Altura uterina & $45(42,9)$ & $7(6,8)$ & $53(50,5)$ & Ruim \\
Apresentação & $74(70,5)$ & $17(16,2)$ & $14(13,3)$ & Ruim \\
Batimentos Cardiofetais & $73(69,5)$ & $2(1,9)$ & $30(28,6)$ & Ruim \\
Movimento fetal & $59(56,2)$ & $8(7,6)$ & $38(36,2)$ & \\
\hline
\end{tabular}

Fonte: Elaboração própria.

Nota: Sinal convencional utilizado:

- Dado numérico igual a zero não resultante de arredondamento. 
$\mathrm{Na}$ seção de Exames Complementares de Rotina predominou itens com registro bom, sendo esta a seção com melhores resultados.
A completude variou entre 61,0\% (ruim) e 94,3\% (bom), referentes ao exame de urocultura e glicemia em jejum, respectivamente (Tabela 4).

Tabela 4-Completude dos registros dos exames complementares de rotina nas cadernetas da gestante. São Luís, Maranhão, Brasil - maio 2017-set. 2018 (N=105)

\begin{tabular}{|c|c|c|c|}
\hline Variáveis & $\begin{array}{c}\text { Sim } \\
\text { n (\%) }\end{array}$ & $\begin{array}{c}\text { Não } \\
\text { n (\%) }\end{array}$ & Classificação \\
\hline \multicolumn{4}{|l|}{ Há registro quanto à (ao): } \\
\hline Tipagem Sanguínea e Fator Rh & $98(93,3)$ & $7(6,7)$ & Bom \\
\hline Glicemia em jejum & $99(94,3)$ & $6(5,7)$ & Bom \\
\hline Sífilis (teste rápido) e/ou VDRL* & $98(93,3)$ & $7(6,7)$ & Bom \\
\hline $\mathrm{HIV}^{* *} /$ Anti-HIV & $95(90,5)$ & $10(9,5)$ & Bom \\
\hline Hepatite B - HbsAg**** & $95(90,5)$ & $10(9,5)$ & Bom \\
\hline Toxoplasmose & $95(90,5)$ & $10(9,5)$ & Bom \\
\hline Hemoglobina/Hematócrito & $94(89,5)$ & $11(10,5)$ & Regular \\
\hline Urina Tipo I & $93(88,6)$ & $12(11,4)$ & Regular \\
\hline Urocultura & $64(61,0)$ & $41(39,1)$ & Ruim \\
\hline Ultrassonografia (opcional) & $97(92,4)$ & $8(7,6)$ & Bom \\
\hline
\end{tabular}

Fonte: Elaboração própria.

Notas:

* VDRL: Venereal Disease Research Labotatory.

** HIV: Vírus da Imunodeficiência Humana.

*** HbsAg: Antígeno de Superfície do Vírus da Hepatite B.

A completude de todos os registros da seção de Imunização foi classificada como ruim. Verificou-se que 20,0\% das cadernetas encontravam-se sem registros de vacina antitetânica, vacina hepatite B e vacina influenza, e $28,6 \%$ encontravam-se sem registro de vacina Difteria, Tétano e Coqueluche (dTpa) (dados não apresentados em tabela). Na seção de Atividades Complementares, todos os componentes obtiveram registro muito ruim, correspondendo à seção com pior avaliação (Tabela 5).

Tabela 5 - Completude dos registros de atividades complementares nas cadernetas da gestante. São Luís, Maranhão, Brasil - maio 2017-set. 2018 (N=105)

\begin{tabular}{|c|c|c|c|c|}
\hline Variáveis & $\begin{array}{c}\text { Sim } \\
\text { n }(\%)\end{array}$ & $\begin{array}{c}\text { Não } \\
\text { n }(\%)\end{array}$ & $\begin{array}{c}\text { Parcial } \\
\text { n }(\%)\end{array}$ & Classificação \\
\hline \multicolumn{5}{|c|}{ Há registro quanto à (ao): } \\
\hline Atividades educativas & $11(10,5)$ & $94(89,5)$ & - & Muito ruim \\
\hline Visita à maternidade & $2(1,9)$ & $103(98,1)$ & - & Muito ruim \\
\hline Consulta odontológica & $3(2,9)$ & $102(97,1)$ & - & Muito ruim \\
\hline Pré-natal do parceiro* & $0(0,0)$ & $88(83,8)$ & $4(3,8)$ & Muito ruim \\
\hline \multicolumn{5}{|l|}{ Fonte: Elaboração própria. } \\
\hline $\begin{aligned} \text { Notas: Sinal convencional utiliz } \\
\text { - Dado numérico igual a } \\
\text { * Excluídas } 13 \text { caderneta }\end{aligned}$ & don & O. & & \\
\hline
\end{tabular}

Ao realizar a classificação da completude de cada caderneta da gestante, observou-se que 20,0\% foram consideradas com registro muito ruim, $72,4 \%$ com registro ruim e 7,6\% com registro regular. Destaca-se que nenhuma caderneta foi classificada com registro bom ou excelente. 


\section{Discussão}

Por se tratar de uma ferramenta capaz de permitir uma intercomunicação entre os profissionais de assistência à mulher ao longo da gestação, parto e puerpério, a legibilidade e completude dos registros na caderneta da gestante são fundamentais. A baixa legibilidade e a incompletude podem comprometer o adequado acompanhamento, pois ocasiona dificuldades de interpretação e análise das informações pelos diferentes profissionais que prestam assistência à mulher no período gravídico-puerperal ${ }^{(10)}$. A dificuldade de interpretação dos dados pode levar à baixa confiabilidade dos profissionais em relação às informações registradas, podendo ocasionar repetições desnecessárias de procedimentos e/ou questionamentos $^{(7)}$.

Algumas propostas sugerem a informatização da caderneta da gestante, com a criação de um sistema de informação integrado, que proporcione o registro e o levantamento de dados em qualquer unidade em que a gestante seja atendida. Um dos aspectos positivos dessa proposta é a boa legibilidade dos registros, entretanto, a informatização não garante a boa qualidade do preenchimento dos dados. Portanto, independente de ser digital ou manual, cabe aos profissionais de saúde o esforço para o adequado registro das informações, de forma fidedigna e completa $^{(14-15)}$.

Nesta pesquisa, o registro do nome da gestante correspondeu ao componente da caderneta com melhor avaliação, sendo considerado com registro excelente. No entanto, os resultados encontrados mostraram um registro muito ruim para o item "como gosta de ser chamada". Diante disso, cabe ressaltar que conhecer a forma como a gestante gosta de ser chamada pode gerar um ambiente agradável e constituir um dos elementos importantes para o estabelecimento de vínculo ${ }^{(16)}$.

Estudos mostram que o vínculo é resultante de bom acolhimento, que se traduz por gestos simples, como tratá-la cordialmente, ouvi-la com atenção e chamá-la pelo nome, especialmente pela forma como gosta de ser chamada.

O vínculo alcançado na relação entre profissionais e gestantes tem sido considerado uma forma de garantir a adesão e o sucesso do acompanhamento pré-natal ${ }^{(16-18)}$. Sendo assim, tendo em vista que esse componente da caderneta constitui um elemento que pode favorecer uma formação de vínculo, torna-se importante a valorização do registro dessa informação.

Dentre os registros da seção de Classificação de Risco Gestacional com menores níveis de completude está a informação relativa ao planejamento da gravidez. Uma gravidez não planejada é aquela que não foi programada pelo casal ou pela mulher, podendo ser indesejada (quando se contrapõe aos seus desejos e expectativas) ou inoportuna (quando ocorre em momento considerado desfavorável). Ambas podem estar associadas a um conjunto de agravos relacionados à saúde materna e perinatal ${ }^{(19-20)}$. A ocorrência de uma gravidez não planejada apresenta um risco aumentado para a realização de abortamento, elevando, por conseguinte, o risco de morbidade e mortalidade materna. Além disso, esse fenômeno pode representar um risco aumentado de ansiedade e depressão, especialmente no período pós-parto ${ }^{(19-21)}$.

Estudo realizado com 2.557 gestantes no sul do país verificou que $65,0 \%$ delas não planejaram a gravidez ${ }^{(21)}$. Outro estudo, realizado com 1.121 mulheres grávidas em um município do nordeste brasileiro, demonstrou uma frequência de $60,2 \%$ para gravidez não pretendida ${ }^{(22)}$. Tais resultados revelam uma grande ocorrência desse fenômeno, o que indica que a investigação e o registro dessa informação não devem ser negligenciados, devido às implicações que pode ocasionar para a saúde materno-infantil.

A seção referente às Atividades Complementares foi a que obteve pior avaliação. Nessa seção, o registro referente às ações educativas apresentou o melhor resultado, mas, ainda assim, com nível muito baixo de completude, inferior ao observado em outros estudos. Em Recife, por exemplo, estudo verificou que 37,0\% dos cartões continham registro de, pelo menos, uma atividade educativa ${ }^{(23)}$.

Pesquisa desenvolvida com o objetivo de conhecer a percepção de gestantes acerca da educação em saúde destacou alguns fatores que 
estimulam a participação nas atividades educativas, como os temas abordados e o esclarecimento de dúvidas. Por outro lado, dentre os entraves para a participação, foram mencionados a pouca divulgação pelos profissionais de saúde e o desestímulo à participação por parte dos familiares $^{(24)}$. Resta, portanto, conhecer os motivos que ocasionaram o preenchimento muito ruim desse componente: se a caderneta não foi utilizada para o registro de realização das ações educativas, se essas ações não foram realizadas ou se foram pouco divulgadas, ou ainda, se não foram valorizadas pelas gestantes e/ou familiares.

Um registro muito ruim também foi observado para "visita à maternidade". Diante disso, destaca-se que, desde 2007, toda gestante tem o direito, garantido pela Lei n. 11.634, de conhecer e visitar previamente a maternidade onde será realizado seu parto. Essa vinculação deve ser assegurada no ato de sua inscrição no programa de assistência pré-natal ${ }^{(2)}$. Nesse contexto, a caderneta da gestante aparece como um instrumento que permite aos profissionais da atenção pré-natal o registro do nome do serviço de saúde indicado para o parto e o registro da realização de visita prévia a esse serviço ${ }^{(5)}$. Contudo, os resultados encontrados mostram uma completude muito ruim para ambas as informações.

Em âmbito nacional, pesquisa evidenciou que $41,3 \%$ das mulheres entrevistadas não foram orientadas sobre a maternidade de referência e que $16,2 \%$ procuraram mais de um serviço para admissão para o parto ${ }^{(25)}$. Nesta pesquisa, 47,6\% das cadernetas apresentaram o registro do serviço de saúde indicado para o parto, sugerindo que esse percentual de gestantes recebeu essa informação, embora algumas gestantes possam ter sido orientadas e a informação não registrada.

Segundo o Ministério da Saúde ${ }^{(26)}$, a vinculação da gestante ao local onde será realizado o seu parto é uma ação fundamental para que ela sinta segurança no momento do nascimento do bebê, para evitar a peregrinação em busca de vaga, situação que pode colocar em risco a vida da mulher e da criança. Diante disso, a unidade de assistência ao pré-natal deve favorecer a visita das gestantes à maternidade de referência, fortalecendo assim o vínculo de confiança entre a mulher e o serviço.

O componente da seção de Atividades Complementares com pior avaliação foi referente ao pré-natal do parceiro, que apresentou nenhum registro completo. A assistência pré-natal, até pouco tempo, apresentava como foco somente a mãe e o bebê. Atualmente, a estratégia pré-natal do parceiro tem incentivado a inserção e o envolvimento do homem nesse contexto, especialmente com o objetivo de prepará-lo para uma paternidade ativa e consciente, compartilhando com a mulher os cuidados com o filho. Além disso, essa estratégia objetiva a detecção precoce de doenças, a atualização vacinal e o estímulo à participação do homem em atividades educativas ${ }^{(5,27)}$.

A inserção de um espaço para o pré-natal do parceiro na caderneta da gestante constituiu uma importante medida para o estímulo à sua realização. No entanto, a constatação dos baixos níveis de preenchimento desse espaço sugere uma incipiente utilização dessa estratégia. A literatura refere que, por mais simples que pareça estender a assistência pré-natal ao parceiro, os serviços de saúde ainda encontram dificuldades nessa inserção. Em alguns casos, a conscientização profissional e a sensibilização das gestantes e seus parceiros ainda se fazem necessárias ${ }^{(28)}$.

Por outro lado, dentre os principais motivos para a ausência do parceiro no acompanhamento pré-natal foi informado a falta de tempo devido ao trabalho; ainda inexistem estratégias para superar essa dificuldade. Dessa forma, autores sugerem a promoção de espaços de diálogo e troca de experiências em horários que permitam a participação do parceiro e, ainda, a discussão acerca da importância de leis trabalhistas que possam garantir sua inclusão na rotina pré-natal, sem prejuízos para o trabalho, permitindo a emissão de atestado de acompanhante nesse período ${ }^{(28-29)}$.

No geral, o preenchimento das informações nas cadernetas analisadas foi ruim. Esses resultados demonstram uma subutilização desse instrumento, o que pode resultar em prejuízos para o acompanhamento da mulher no ciclo gravídico-puerperal, dificultando a análise da 
evolução da gravidez e a intercomunicação entre os profissionais que prestam assistência a essa mulher. Além disso, partindo do pressuposto de que a qualidade dos registros efetuados pode ser um reflexo da qualidade da assistência, os resultados encontrados denotam que a qualidade da atenção pré-natal precisa ser melhorada em muitos aspectos, especialmente no que se refere aos componentes atualmente inseridos nesse instrumento.

Contudo, ainda existe a interrogação se a lacuna está na assistência ou nos registros, tendo em vista que alguns procedimentos podem estar sendo realizados e não registrados, o que indica a necessidade de conscientização dos profissionais de saúde para a valorização da caderneta da gestante.

\section{Conclusão}

A maioria das cadernetas da gestante apresentaram registros legíveis, no entanto, incompletos. Constatou-se que foram priorizados os registros referentes ao nome da gestante, data de nascimento, dados quantitativos relacionados às gestações anteriores, data da última menstruação, data provável do parto e a maioria dos exames de rotina. Os registros mais ausentes foram relativos ao pré-natal do parceiro, visita à maternidade, consulta odontológica, número do Sisprenatal, gráfico de acompanhamento nutricional, curva de altura uterina por idade gestacional, entre outros.

Conclui-se que os registros nas cadernetas da gestante foram predominantemente ruins, denotando uma desvalorização desse instrumento e um provável descumprimento das recomendações do Ministério da Saúde para uma assistência de qualidade.

Com a implantação da caderneta da gestante ocorreu um incremento de informações e procedimentos a serem registrados. Essa maior demanda por registros pode justificar os baixos níveis de completude verificados nesta pesquisa. Outros fatores que podem estar influenciando essa subutilização são o desconhecimento da finalidade e da importância dessa ferramenta pelos profissionais de saúde e a grande demanda por atendimento, sendo necessários estudos que corroborem essas proposições, a fim de propiciar intervenções oportunas.

Vale destacar que, concomitante à implantação da caderneta, foi publicado o manual da caderneta da gestante para profissionais de saúde e, para iniciar sua implementação, deveria ser realizado um treinamento. Sendo assim, sugere-se a realização de auditorias e capacitações periódicas dos profissionais da assistência pré-natal, no intuito de promover sensibilização para a adequada utilização dessa ferramenta, contribuindo para melhoria na qualidade dos registros.

\section{Colaborações}

1 - concepção, projeto, análise e interpretação dos dados: Thaíse Almeida Rodrigues e Lena Maria Barros Fonseca;

2 - redação do artigo e revisão crítica relevante do conteúdo intelectual: Thaíse Almeida Rodrigues, Ana Karina Bezerra Pinheiro, Andressa Arraes Silva, Luciene Rocha Garcia Castro, Milka Borges da Silva e Lena Maria Barros Fonseca;

3 - aprovação final da versão a ser publicada: Thaíse Almeida Rodrigues, Ana Karina Bezerra Pinheiro, Andressa Arraes Silva, Luciene Rocha Garcia Castro, Milka Borges da Silva e Lena Maria Barros Fonseca.

\section{Referências}

1. Huang A, Wu K, Zhao W, Hu H, Yang Q, Chen D. Attendance at prenatal care and adverse birth outcomes in China: A follow-up study based on Maternal and Newborn's Health Monitoring System. Midwifery. 2018;57:26-31. DOI: 10.1016/j. midw.2017.10.018

2. Brasil. Ministério da Saúde. Secretaria de Atenção à Saúde. Departamento de Atenção Básica. Atenção ao pré-natal de baixo risco [Internet]. Brasília (DF); 2012. Cadernos de Atenção Básica, n. 32 [cited 2018 Out 2]. Available from: http://bvsms.saude. gov.br/bvs/publicacoes/cadernos_atencao_ basica_32_prenatal.pdf 
3. Polgliani RBS, Santos Neto ET, Zandonade E. Informações dos cartões de gestante e dos prontuários da atenção básica sobre assistência prénatal. Rev Bras Ginecol Obstet. 2014;36(6):269-75. DOI: $10.1590 /$ S0100-720320140004907

4. Brasil. Ministério da Saúde. Secretaria de Atenção à Saúde. Departamento de Ações Programáticas Estratégicas. Coordenação Geral de Saúde das Mulheres. Nota Informativa. Assunto: Distribuição da Caderneta da Gestante [Internet]. Brasília (DF); 2015 [cited 2018 Out 2]. Available from: http://189.28.128.100/dab/docs/portaldab/ documentos/nota_informativa_distribuicao.pdf .

5. Brasil. Ministério da Saúde. Caderneta da gestante [Internet]. 4a ed. Brasília(DF); 2018 [cited 2018 Out 2]. Available from: https://portalarquivos2.saude.gov. br/images/pdf/2018/agosto/31/Caderneta-daGestante-2018.pdf

6. Donabedian A. The quality of care. How can it be assessed? JAMA. 1988;260(12):1743-48. DOI: 10.1001/jama.260.12.1743

7. Santos TMMG, Abreu APSB, Campos TG. Avaliação dos registros no cartão de prénatal da gestante. Rev enferm UFPE on line. 2017;11(Suppl 7):2939-45. DOI: 10.5205/reuol.1100798133-3-SM.1107sup201715

8. Donabedian A. La Calidad de la Atención Médica: Definición y métodos de evaluación. México: La Prensa Médica Mexicana; 1984.

9. Santos Neto ET, Oliveira AE, Zandonade E, Gama SGN, Leal MC. O que os cartões de prénatal das gestantes revelam sobre a assistência nos serviços do SUS da Região Metropolitana da Grande Vitória, Espírito Santo, Brasil? Cad Saúde Pública. 2012;28(9):1650-62. DOI: https://doi. org/10.1590/S0102-311X2012000900005

10. Coêlho TTG, Medeiros ACQ, Ribeiro WCS, Menêzes TB. Avaliação do grau de completude do cartão da gestante de puérperas atendidas em um hospital universitário. Rev bras ciênc saúde. 2015;19(2):117-22.DOI:10.4034/RBCS.2015.19.02.05

11. Zanchi M, Gonçalves CV, Cesar JA, Dumith SC. Concordância entre informações do Cartão da Gestante e do recordatório materno entre puérperas de uma cidade brasileira de médio porte. Cad Saúde Pública. 2013;29(5):1019-28. DOI: https:// doi.org/10.1590/S0102-311X2013000500019

12. Romero DE, Cunha CB. Avaliação da qualidade das variáveis sócio-econômicas e demográficas dos óbitos de crianças menores de um ano registrados no Sistema de Informações sobre Mortalidade do Brasil (1996/2001). Cad Saúde Pública. 2006;22(3):673-84. DOI: https://doi.org/ 10.1590/S0102-311X2006000300022

13. Romero DE, Cunha CB. Avaliação da qualidade das variáveis epidemiológicas e demográficas do Sistema de Informações sobre Nascidos Vivos, 2002. Cad Saúde Pública. 2007;23(3):701-14. DOI: https:// doi.org/10.1590/S0102-311X2007000300028

14. Santos Neto ET, Leal MC, Oliveira AE, Zandonade E, Gama SGN. Concordância entre informações do Cartão da Gestante e da memória materna sobre assistência pré-natal. Cad Saúde Pública. 2012;28(2):256-66. DOI: https://doi.org/10.1590/ S0102-311X2012000200005

15. Conceição MC, Moura LKB, Monte TL, Landim CA, Matos FTC, Ferraz MAAL. Desenvolvimento de um programa para computador de acompanhamento de gestantes [Internet]. Espacios. 2017 [cited 2018 Out 2];38(58):21-9. Available from: https://www. revistaespacios.com/a17v38n58/a17v38n58p21. pdf

16. Pio DAM, Capel MS. Os significados do cuidado na gestação [Internet]. Rev Psicol Saúde. 2015 [cited 2018 Out 2];7(1):74-81. Available from: http:// pepsic.bvsalud.org/pdf/rpsaude/v7n1/v7n1a10. pdf

17. Silva MZN, Andrade AB, Bosi MLM. Acesso e acolhimento no cuidado pré-natal à luz de experiências de gestantes na Atenção Básica. Saúde Debate. 2014;38(103):805-16. DOI: 10.5935/ 0103-1104.20140073

18. Alves CN, Wilhelm LA, Barreto CN, Santos CC, Meincke SMK, Ressel LB. Cuidado pré-natal e cultura: uma interface na atuação da enfermagem. Esc Anna Nery. 2015;19(2):265-71. DOI: 10.5935/ $1414-8145.20150035$

19. Langer A. El embarazo no deseado: impacto sobre la salud y la sociedad en América Latina y el Caribe [Internet]. Rev Panam Salud Pública. 2002 [cited 2018 Out 2];11:192-203. Available from: https:// www.scielosp.org/pdf/rpsp/2002.v11n3/192-205/es

20. Gipson JD, Koenig MA, Hindin MJ. The effects of unintended pregnancy on infant, child, and parental health: a review of the literature. Stud Fam Plann. 2008;39(1):18-38. DOI: 10.1111/j.1728-4465.2008.00148.x

21. Prietsch SOM, Gonzalez-Chica DA, Cesar JA, Mendoza-Sassi RA. Gravidez não planejada no extremo Sul do Brasil: prevalência e 
fatores associados. Cad Saúde Pública. 2011; 27(10):1906-16. DOI: https://doi.org/10.1590/ S0102-311X2011001000004

22. Brito CNO, Alves SV, Ludermir AB, Araújo TVB. Depressão pós-parto entre mulheres com gravidez não pretendida. Rev Saúde Pública. 2015;49:33. DOI: $10.1590 /$ S0034-8910.2015049005257

23. Barreto FDFP, Albuquerque RM. Discrepâncias entre o informe verbal e os registros no cartão da gestante, um instrumento negligenciado. Rev Bras Ginecol Obstet. 2012;34(6):259-67. DOI: https:// doi.org/10.1590/S0100-72032012000600004

24. Souza VB, Roecker S, Marcon SS. Ações educativas durante a assistência pré-natal: percepção de gestantes atendidas na rede básica de Maringá-PR. Rev Eletr Enf. 2011;13(2):199-210. DOI: http:// dx.doi.org/10.5216/ree.v13i2.10162

25. Viellas EF, Domingues RMSM, Dias MAB, Gama SGN, Theme Filha MM, Costa JV, et al. Assistência pré-natal no Brasil. Cad. Saúde Pública. 2014;30(Suppl 1):S85-S100. DOI: https://doi. org/10.1590/0102-311X00126013

26. Brasil. Ministério da Saúde. Secretaria de Atenção à Saúde. Gravidez, parto e nascimento com saúde, qualidade de vida e bem-estar [Internet].
Brasília (DF); 2013 [cited 2018 Out 5]. Available from: http://bvsms.saude.gov.br/bvs/publicacoes/ gravidez_parto_nascimento_saude_qualidade.pdf

27. Brasil. Ministério da Saúde. Secretaria de Atenção à Saúde. Departamento de Ações Programáticas Estratégicas. Coordenação Nacional de Saúde do Homem. Guia do Pré-natal do Parceiro para Profissionais de Saúde [Internet]. Rio de Janeiro (RJ); 2016 [cited 2018 Out 2]. Available from: https://portalarquivos2.saude.gov.br/images/ pdf/2016/agosto/11/guia_PreNatal.pdf

28. Cardoso VEPS, Silva Junior AJ, Bonatti AF, Santos GWS, Ribeiro TAN. A participação do parceiro na rotina pré-natal sob a perspectiva da mulher gestante. Rev Fund Care Online. 2018; 10(3):856-62. DOI:http://dx.doi.org/10.9789/2175-5361.2018.v10i3. 856-862

29. Henz GS, Medeiros CRG, Salvadori M. A inclusão paterna durante o pré-natal. Rev Enferm Atenção Saúde. 2017;6(1):52-66. DOI: https://doi. org/10.18554reas.v6i1.2053

Recebido: 7 de janeiro de 2020

Aprovado: 4 de março de 2020

Publicado: 9 de abril de 2020

A Revista Baiana de Enfermagem utiliza a Licença Creative Commons - Atribuição-NãoComercial 4.0 Internacional. https://creativecommons.org/licenses/by-nc/4.0/

Este artigo é de acesso aberto distribuído sob os termos da Licença Creative Commons (CC BY-NC). Esta licença permite que outros remixem, adaptem e criem a partir do seu trabalho para fins não comerciais. Embora os novos trabalhos tenham de lhe atribuir o devido crédito e não possam ser usados para fins comerciais, os usuários não têm de licenciar esses trabalhos derivados sob os mesmos termos. 